\title{
Transfer matrix modeling and experimental validation of cellular porous material with resonant inclusions
}

\author{
Olivier Doutres ${ }^{\text {a) }}$ and Noureddine Atalla \\ Groupe d'acoustique de l'Université de Sherbrooke GAUS, Department of Mechanical Engineering, \\ Université de Sherbrooke (Qc), J1K 2Rl, Canada \\ Haisam Osman \\ Highlands Ranch, Colorado 80130, USA
}

(Received 6 November 2014; revised 17 March 2015; accepted 19 April 2015)

\begin{abstract}
Porous materials are widely used for improving sound absorption and sound transmission loss of vibrating structures. However, their efficiency is limited to medium and high frequencies of sound. A solution for improving their low frequency behavior while keeping an acceptable thickness is to embed resonant structures such as Helmholtz resonators (HRs). This work investigates the absorption and transmission acoustic performances of a cellular porous material with a two-dimensional periodic arrangement of HR inclusions. A low frequency model of a resonant periodic unit cell based on the parallel transfer matrix method is presented. The model is validated by comparison with impedance tube measurements and simulations based on both the finite element method and a homogenization based model. At the HR resonance frequency (i) the transmission loss is greatly improved and (ii) the sound absorption of the foam can be either decreased or improved depending on the HR tuning frequency and on the thickness and properties of the host foam. Finally, the diffuse field sound absorption and diffuse field sound transmission loss performance of a $2.6 \mathrm{~m}^{2}$ resonant cellular material are measured. It is shown that the improvements observed at the Helmholtz resonant frequency on a single cell are confirmed at a larger scale.
\end{abstract}

(C) 2015 Acoustical Society of America. [http://dx.doi.org/10.1121/1.4921027]

[OU]

Pages: $3502-3513$

\section{INTRODUCTION}

For many years, research has been oriented toward the improvement of the low frequency efficiency of passive sound absorbing materials. Such materials, constituted of a fluid phase saturating an open porous solid matrix, dissipate acoustic energy by visco-thermal interactions between the two phases and are efficient for frequencies with wavelengths in air on the order of magnitude of the material thickness. A low frequency solution is to embed Helmholtz resonators (HRs) in the porous matrix. One of the first works describing such structure is a patent awarded to Borchers et al. ${ }^{1}$ in which a resonant acoustic protection is proposed to attenuate low frequency noise level inside payload fairings of launch vehicles. This resonant structure aimed at improving classical sound packages used for this application. The latter are usually made of a homogeneous blanket or a multilayer including heavy layers. ${ }^{2}$ Much later, Sugie et al. ${ }^{3}$ proposed a similar heterogeneous material made of fibrous sound absorbers with resonant inclusions in order to improve the low frequency sound transmission loss of double-leaf structures at the mass-air-mass resonance frequency. According to the authors of Ref. 3, their idea stems from the works of Mason and Fahy, ${ }^{4}$ Prydz et al., ${ }^{5}$ and Kuntz et al., ${ }^{6}$ where the HRs where attached directly on the panel. Sugie et $a l^{3}$ suggested that including the resonant element within the fibrous sound package in between the two panels allows

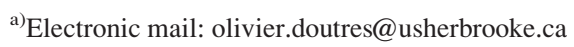

for a more flexible optimization of the HR effect and a low frequency sound insulation improvement. More recently, the acoustic community had shown a keen interest in this type of resonant structure. The equivalent material (also called metacomposite or metamaterial) presents a negative bulk modulus at the HR resonance frequency and in consequence blocks sound at this frequency. ${ }^{7-11}$ Most of these works mainly focused on the transmission properties of the resonant material and highlighted the large transmission dip at the HR resonance frequency. ${ }^{7-9}$ Boutin and Bécot, ${ }^{11}$ Lagarrigue et al., ${ }^{12}$ and Groby et al. ${ }^{13}$ recently investigated the sound absorption efficiency of rigid backed acoustic foams with resonant split hollow cylinder or HR inclusions and showed that it is greatly improved for frequencies with a wavelength much larger than the material thickness.

Under the assumption that the HR periodicity is much smaller than the acoustic wavelength, the resonant materials are usually modeled as homogenized equivalent materials with effective bulk modulus to account for the presence of the resonant inclusion. ${ }^{7-11,14}$ Fang et al. ${ }^{14}$ developed an analytical expression of the equivalent bulk modulus of a metamaterial made of a one-dimensional (1D) array of HRs flush mounted on an acoustic duct. Boutin and Bécot ${ }^{10,11}$ developed a similar expression for resonant material made of HRs embedded in a foam matrix with a three-dimensional (3D) periodicity using the homogenization method. In the case of cellular resonant material with a two-dimensional (2D) periodicity, Lagarrigue et $a l .{ }^{12}$ and Groby et al. ${ }^{13}$ show that the sound absorption behavior of the material can be impacted by the orientation of 
the resonator aperture. Lagarrigue et al. ${ }^{12}$ proposed an interesting 2D semi-analytical model in the case of embedded slit rings in a large periodic foam unit cell for which the slit orientation relative to the rigid backing is taken into account. This model performs well at low frequencies but is restricted to $2 \mathrm{D}$ and to the rigid backing configuration. More complex structures such as a single wall or a double wall treated with a classical homogeneous or multilayer sound package are usually modeled by the transfer matrix method (TMM). ${ }^{15,16} \mathrm{TMM}$ is adequate for describing layered partitions, composed of infinite plates and porous layers, with acceptable accuracy. ${ }^{17}$ Verdière et al. ${ }^{18,19}$ recently provided an extension of the TMM to account for the assembly of materials in parallel and series. It is referred to as the parallel transfer matrix method (P-TMM) in Ref. 19. The latter work thus opens the door for TMM modeling of resonant inclusions.

The work presented in this paper deals with the P-TMM modeling and the experimental validation of cellular resonant porous material made of periodic unit-cell (PUC) with a spherical HR inclusion arranged in a 2D periodicity. The HR lies in the center of the PUC and can be either completely or partially embedded in the foam. Partial embedment in this work refers to PUCs for which the HR is inserted in the host foam but with the neck aperture in contact with the surrounding air medium. This configuration ensures a strong influence of the HR at its resonance frequency even if it is embedded in a high flow resistivity foam. ${ }^{13}$ The P-TMM modeling presented in this paper allows such flexibility on the description of the main PUC geometrical characteristics since it is based on the combination of porous element in parallel and series to account, respectively, for the HR inclusion and its potential coverings. Both the normal incidence sound absorption and sound transmission loss behaviors are investigated and focus is put on the effect of the HR orientation within the foam matrix.

This paper is organized as follows. In Sec. II, the P-TMM model of the PUC is presented. This section also briefly presents two existing models used to validate the proposed P-TMM approach: the homogenization model and the finite element (FE) model. The experimental setups, foam substrate, and resonant materials are described in Sec. III. In Sec. IV A, P-TMM predictions of the normal incidence acoustical behavior of a resonant PUC made of a HR fully embedded in low flow resistivity open-cell foam are validated by comparison with both the homogenization and FE models. The proposed P-TMM model is then validated on a PUC made of a HR partially embedded in medium flow resistivity melamine foam by comparison with numerical and experimental results. Finally, measurements conducted on a $2.6 \mathrm{~m}^{2}$ resonant material made of $156 \mathrm{HRs}$ are presented in Sec. IV B. Diffuse field sound transmission loss and sound absorption measurements were carried out to corroborate the HR contributions observed in the 1D analysis.

\section{THEORY}

\section{A. P-TMM model}

The proposed sound package is defined as a $2 \mathrm{D}$ arrangement of cubic PUCs of dimension $l \times L \times L$ with $l \approx L$ $\approx 100 \mathrm{~mm}$; as shown in Figs. 1(a) and 1(b). The PUC presented in Fig. 1 is composed of a HR fully embedded in a porous substrate. The P-TMM (Refs. 16-19) is used to model its 1D acoustical behavior. In this approach, the PUC is modeled as a stack in series of three layers of thickness $l_{1}$, $l_{2}$, and $l_{3}$ [see Fig. 1(c)]. Layers 1 and 3 are homogeneous foams while layer 2 (the middle layer) is a parallel assembly of the HR and the foam matrix [see Fig. 1(d)]. The three layers can be made of different porous materials. In this paper, layer 1 is optional and will only be used when ones want to completely bury the HR: material for which layer 1 is present (respectively, missing) are referred to as PUC with fully (respectively, partially) embedded HR.

The transfer matrix of the PUC presented in Fig. 1(c) is given by

$$
T^{\mathrm{PUC}}=\left[\begin{array}{ll}
T_{11}^{\mathrm{PUC}} & T_{12}^{\mathrm{PUC}} \\
T_{21}^{\mathrm{PUC}} & T_{22}^{\mathrm{PUC}}
\end{array}\right]=T^{\mathrm{layer}, 1} \times T^{\mathrm{layer}, 2} \times T^{\mathrm{layer}, 3} .
$$

All porous materials are modeled using the classical Johnson-Champoux-Allard equivalent fluid model $1^{16,20,21}$ and thus can be represented by a $2 \times 2$ transfer matrix. The

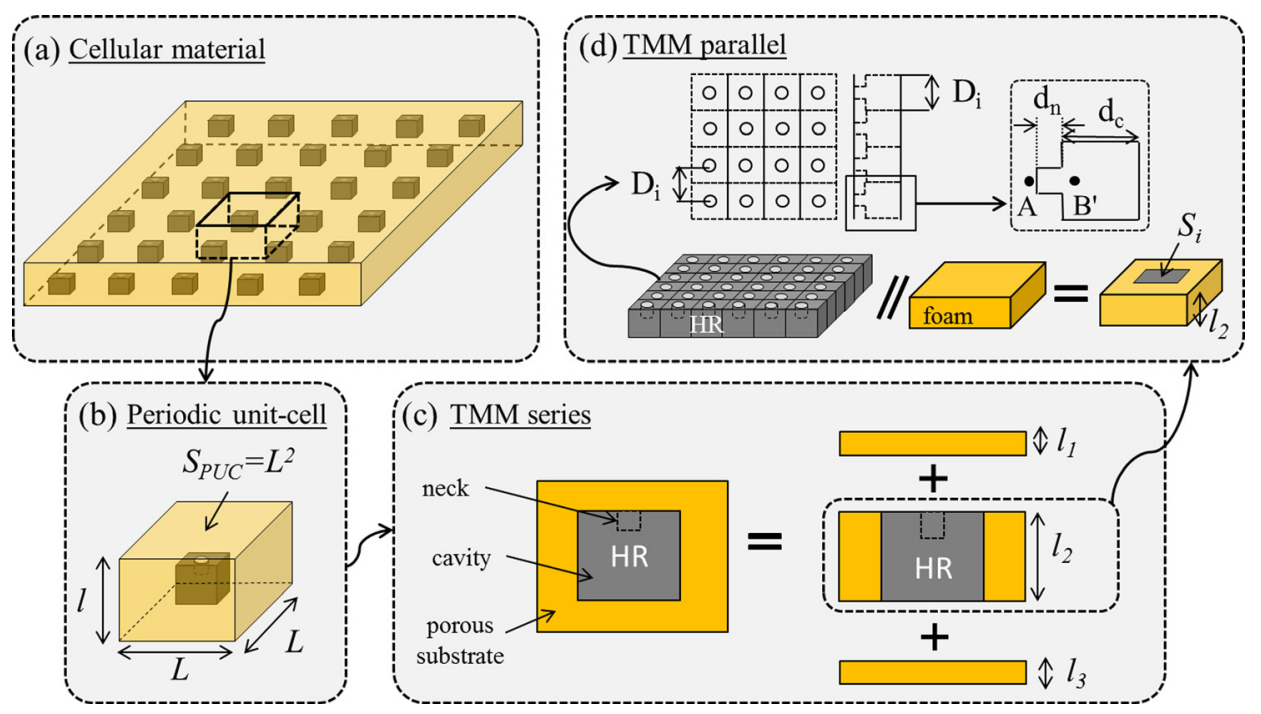

FIG. 1. (Color online) Scheme of the sound package and the PUC. 
transfer matrices of the two outer homogeneous porous layers $T^{\text {layer, } i}$ (with $i=1$ and 3 ) are well known and equal to

$$
\begin{aligned}
T^{\text {layer }, i} & =\left[\begin{array}{ll}
T_{11}^{i} & T_{12}^{i} \\
T_{21}^{i} & T_{22}^{i}
\end{array}\right] \\
& =\left[\begin{array}{cc}
\cos \left(k_{\mathrm{eq}, i} l_{i}\right) & j Z_{\mathrm{eq}, i} \sin \left(k_{\mathrm{eq}, i} l_{i}\right) \\
j \sin \left(k_{\mathrm{eq}, i} l_{i}\right) / Z_{\mathrm{eq}, i} & \cos \left(k_{\mathrm{eq}, i} l_{i}\right)
\end{array}\right],
\end{aligned}
$$

with $j^{2}=-1, k_{\text {eq }, i}$, and $Z_{\text {eq }, i}$ the wave number and characteristic impedance of the equivalent fluid, respectively. These properties are derived from the dynamic density $\rho_{i}$ and dynamic bulk modulus $K_{i}$ of the fluid phase such as $Z_{\text {eq }, i}=\left(\rho_{i} \cdot K_{i}\right)^{1 / 2} / \phi$ and $k_{\text {eq }, i}=\omega\left(\rho_{i} / K_{i}\right)^{1 / 2}$, with $\omega$ the angular frequency and $\phi$ the open porosity of the porous material.

The transfer matrix of layer 2 combines two submatrices which are modeled using a parallel assembly as proposed by Verdière et al. ${ }^{18}$ The following conditions must be fulfilled if one want to use P-TMM: (1) only plane waves propagate upstream and downstream of the periodic construction; (2) only normal incidence plane waves propagate in the construction; (3) no pressure diffusion exists between adjacent parallel elements, (4) the wavelength is much larger than the PUC, and (5) each element can be represented by a $2 \times 2$ transfer matrix. Conditions (2)-(5) are fulfilled here since we consider (i) low frequencies (that is frequencies much lower than $c_{0} / L$ ), (ii) porous substrate with intermediate airflow resistivity, (iii) equivalent fluid models for predicting their acoustic behavior and finally, (iv) a surface impedance model to represent the HR. However, assumption (1) seems physically unrealistic around the HR resonance frequency in the vicinity of the HR neck. According to Ref. 18, the transfer matrix of layer 2 can be written in the form

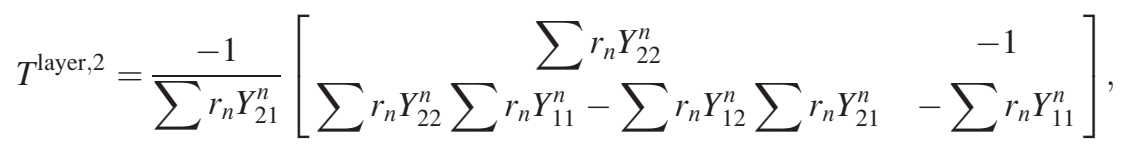

with $Y^{n}$ the components of the admittance matrix for each element; here $n=$ mat for the porous substrate and $n=\mathrm{HR}$ for the resonator element. $r_{n}$ is the surface ratio of each element and is similar to the "percentage open area" for perforated panels. Knowing the surface of the unit cell $S_{\text {PUC }}$ [see Fig. 1(b)] and the surface of the resonator $S_{i}$ [see Fig. 1(d)], thus $r_{\mathrm{HR}}=r=S_{i} / S_{\mathrm{PUC}}$ and $r_{\mathrm{mat}}=1-r_{\mathrm{HR}}$. The admittance matrix for each element is given by

$$
Y^{n}=\left[\begin{array}{cc}
Y_{11}^{n} & Y_{12}^{n} \\
Y_{21}^{n} & Y_{22}^{n}
\end{array}\right]=\frac{1}{T_{12}^{n}}\left[\begin{array}{cc}
T_{22}^{n} & T_{21}^{n} T_{12}^{n}-T_{22}^{n} T_{11}^{n} \\
1 & -T_{11}^{n}
\end{array}\right]
$$

The transfer matrix of the porous matrix $T^{\text {mat }}$ is given by Eq. (2) and the one of the resonator $T^{\mathrm{HR}}$ is given in Sec. II B.

For practical reasons related to material fabrication (see Sec. III), the HRs used in this work are spherical and the inner porous layer (of thickness $l_{2}$ ) is perforated in order to host the spherical HR as shown in Fig. 2(a). The mismatch between the cylindrical perforation and the spherical geometry of the HR inclusion create thin air layers above and below the resonator within the perforation of layer 2 . The volume of these air layers is significant because of the large size of both the PUC and of the inner perforation; the P-TMM model should thus be modified accordingly. Because it is important to model the fact that the HRs neck is not buried within the inner layer 2 unless layer 1 is used, only the air layer below the HR is taken into account as shown in Fig. 2(b). The inner layer 2 is thus split in two parallel assemblies, named $l_{2 a}$ and $l_{2 \mathrm{HR}}$, assembled in series and its matrix and is given by

$$
T^{\text {layer, } 2}=T^{\text {layer }, 2 \mathrm{HR}} \times T^{\text {layer }, 2 a}
$$

$T^{\text {layer,2m }}(m=$ HR or $a$ ) is the matrix of the parallel elements which are open upstream and downstream. $T^{\text {layer,2HR }}$ is written exactly as Eq. (3) and combines the transfer matrix of the porous layer and the one of the HR. The thickness of this sublayer (i.e., $l_{2 \mathrm{HR}}$ ) is set to the thickness of the HR cavity $d_{c}$ being an equivalent cylinder with the same volume as the spherical HR and the circular cross-section area of the perforation. The transfer matrix of the second parallel assembly $T^{\text {layer, } 2 a}$, combines the transfer matrix of the porous layer and the one of an air layer. The thickness of this sublayer is $l_{2 a}=l_{2}-l_{2 \mathrm{HR}}$. The transfer matrix of the sublayer is also

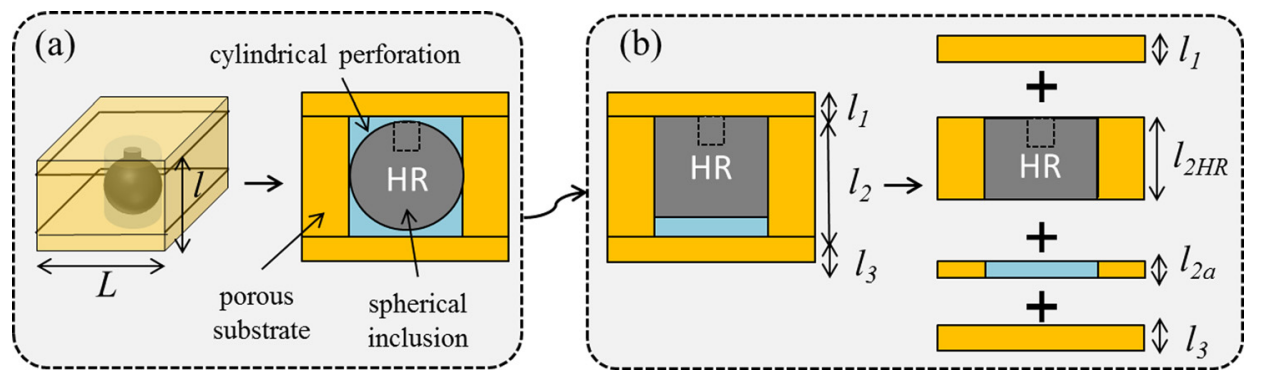

FIG. 2. (Color online) Scheme of the PUC with the perforation and the spherical HR. 
based on Eqs. (3) and (4) but the transfer matrix of the HR is replaced by one of an air layer. The later matrix can be simply calculated from Eq. (2) but $Z_{\mathrm{eq}, i}$ has to be replaced by the characteristic impedance of air $Z_{0}, k_{\mathrm{eq}, i}$ by the wave number in air $k_{0}$ and $l_{i}$ by $l_{2 a}$. Both parallel assemblies $l_{2 a}$ and $l_{2 \mathrm{HR}}$ share the surface ratio of each element $r_{n}$ since they share the same perforation diameter.

\section{B. HR modeling}

The transfer matrix of the resonator $T^{\mathrm{HR}}$ required in Eqs. (2) and (3) for parallel assemblies with resonant inclusion is the one of a resonator array as described in Refs. 16 and 22. It is written as a product of the inertial and acoustic components. $^{22}$ For an acoustic wave impinging on the neck side of the HR (this HR position is called $\mathbf{A B}$ in this paper), its transfer matrix is given by

$$
T^{\mathrm{HR}, \mathrm{AB}}=\left[\begin{array}{cc}
1 & 0 \\
1 / Z_{A} & 1
\end{array}\right]\left[\begin{array}{cc}
1 & j \omega M_{S} \\
0 & 1
\end{array}\right],
$$

with $M_{S}$ the mass per unit area of the resonator array and $Z_{A}$ the acoustic input impedance. Here we limit the study to linear behavior. According to Ref. 16 (see p. 203), the input total impedance of the resonator array under normal incidence plane waves, Fig. 1(d), is given by

$$
\begin{aligned}
Z_{A}= & \frac{1}{s_{e}}\left(Z_{\text {neck }}+Z_{0,0}\left(B^{\prime}\right)\right) \\
= & \frac{1}{s_{e}}\left(\left[\left(\frac{2 d_{n}}{R_{n}}+4\right) R_{s}+j \omega\left(\varepsilon_{i}+\varepsilon_{e}+d_{n}\right) \rho_{0}\right]\right. \\
& \left.-j s_{i} Z_{0} \cot \left(k_{0} d_{c}\right)\right)
\end{aligned}
$$

with $\rho_{O}$ the air density, $c_{O}$ the speed of sound, $k_{O}=\omega / c_{0}$ the wavenumber, $d_{c}$ the resonator cavity thickness, $d_{n}$ the neck's depth, $R_{n}$ the neck's inner radius, $R_{s}$ is a surface resistance equal to $\left(2 \eta \rho_{O} \omega\right)^{1 / 2} / 2, \eta$ the dynamic fluid viscosity, and $s_{e}$ and $s_{i}$ the ratio of the area of the neck aperture $S_{n}$ over the cross-section area of "external" elementary cell and "internal" elementary cell, respectively. The resonator considered in the studied PUC has a negligible wall thickness. The cross-section areas of the internal and external elementary cell are thus taken equal; i.e., $s_{i}=s_{e}=S_{n} / D_{i}{ }^{2}$ with $D_{i}$ shown in Fig. 1(d). Under the large wavelength assumption $\left(\lambda \gg D_{i}\right.$ and $\lambda \gg d_{c}$ ), the normal surface impedance of the air layer at point $B^{\prime}$ is $Z_{0,0}\left(B^{\prime}\right)=-j s_{i} Z_{0} \cot \left(k_{0} d_{c}\right)$. Furthermore, the terms related to the modes of higher order in the resonator cavity are replaced by an added length effect (i.e., $\varepsilon_{i}$ ) added to the resonator neck depth. The shape of the resonator cavity is thus irrelevant and the resonator is characterized by its cavity inner volume. The length of the neck is also corrected (i.e., $\varepsilon_{e}$ ) to account for the mass loading associated to the sound radiation of the neck and to the distortion of the acoustic flow at the resonator surface. In the case of a circular neck aperture in contact with two air media on both ends, the two length corrections can be approximated for $s_{e}<0.16$ by $^{16}$

$$
\varepsilon_{i}=\varepsilon_{e}=0.48 \sqrt{S_{n}}\left(1-1.14 \sqrt{s_{e}}\right)
$$

The resistive part in Eq. (7) [i.e., $\left(2 d_{n} / R_{n}+4\right) R_{s}$ ] accounts for the viscous effects occurring within the resonator neck due to the viscous boundary layer and around its edges at the panel surface due to the distortion of the acoustic flow.

\section{Acoustic properties of the PUC}

Various acoustic features can be determined from the transfer matrix of the PUC given by Eq. (1). It is worth noting that the combination of Eqs. (1), (3), and (6) stands for an acoustic wave impinging on the neck side of the HR (i.e., position AB). The transfer matrix of the PUC in the opposite direction BA, i.e., the PUC is inverted and the acoustic wave now impinges on the rear side of the HR, can be obtained by simply inverting the matrix of Eq. (1) accounting for a change in the sign of the velocity by $-u,{ }^{23}$

$$
T^{\mathrm{PUC}, \mathrm{BA}}=\frac{1}{\operatorname{det}\left(T^{\mathrm{PUC}, \mathrm{AB})}\right.}\left[\begin{array}{cc}
T_{22}^{\mathrm{PUC}, \mathrm{AB}} & T_{12}^{\mathrm{PUC}, \mathrm{AB}} \\
T_{21}^{\mathrm{PUC}, \mathrm{AB}} & T_{11}^{\mathrm{PUC}, \mathrm{AB}}
\end{array}\right] .
$$

The normal incidence sound transmission coefficient $T L_{n}$ is 10 times the common logarithm of the reciprocal of the sound transmission coefficient $\tau$; the latter being the fraction of airborne sound power incident on a material that is transmitted by the material and radiated on the other side. ${ }^{25}$ In the case of an anechoic-backed PUC, it is given by

$$
T L_{n}=-20 \log _{10}\left(\frac{2}{\left|T_{11}^{\mathrm{PUC}}+T_{12}^{\mathrm{PUC}} / Z_{0}+Z_{0} T_{21}^{\mathrm{PUC}}+T_{22}^{\mathrm{PUC}}\right|}\right) .
$$

The sound absorption coefficient $\alpha$ is related to the reflection coefficient $R^{b}$ defined as the ratio of the pressures created by the outgoing and ingoing waves at the surface of the layer: $\alpha=1-\left|R^{b}\right|^{2}$. The normal incidence reflection coefficient of the PUC backed by a rigid backing, $R^{b}$, or by an air cavity of thickness $H, R^{w}$, can be obtained, respectively, by

$$
R^{b}=\frac{T_{11}^{\mathrm{PUC}}-Z_{0} T_{21}^{\mathrm{PUC}}}{T_{11}^{\mathrm{PUC}}+Z_{0} T_{21}^{\mathrm{PUC}}}
$$

and

$$
R^{w}=\frac{T_{11}^{\mathrm{PUC}} T_{11}^{a}+T_{12}^{\mathrm{PUC}} T_{21}^{a}-Z_{0} T_{21}^{\mathrm{PUC}} T_{11}^{a}-Z_{0} T_{22}^{\mathrm{PUC}} T_{21}^{a}}{T_{11}^{\mathrm{PUC}} T_{11}^{a}+T_{12}^{\mathrm{PUC}} T_{21}^{a}+Z_{0} T_{21}^{\mathrm{PUC}} T_{11}^{a}+Z_{0} T_{22}^{\mathrm{PUC}} T_{21}^{a}},
$$

with $T_{i j}{ }^{a}$ the transfer matrix coefficients of the backing cavity which can be obtained from Eq. (2) by replacing $Z_{\mathrm{eq}, i}$ by $Z_{0}, k_{\mathrm{eq}, i}$ by $k_{0}$, and $l_{i}$ by $H$.

\section{Homogenization model}

Boutin $^{10}$ and Boutin and Bécot ${ }^{11}$ recently proposed an analytical model to describe the acoustic wave propagation 
in a porous matrix with embedded HR based on the homogenization method. They defined the "co-dynamic" regime by the coexistence of dynamic phenomena at both micro and macro scales. According to the authors' own words, " $a$ 'co-dynamic' regime requires that the flux induced by the resonating domain is sufficiently small to be negligible at the leading order (so that the effective constitutive parameters are on the order of those of the carrying constituent), but also sufficiently large to contribute to the macroscopic balance." Two important conditions have to be fulfilled for having a co-dynamic regime: (1) the section of the HR neck should be small compared to the section of the PUC so that the velocity in the neck is of the same order as the velocity in the porous matrix and (2) the HR resonance frequency, $f_{0}=\omega_{0} / 2 \pi$, must be tuned in the inertial or visco-inertial regime of the porous matrix in order to fulfill the scale separation required in the homogenization method: $\lambda_{\mathrm{m}}\left(f_{0}\right) /(2 \pi)$ $\gg L$ with $\lambda_{\mathrm{m}}$ the acoustic wavelength in the porous matrix. The latter condition restricts the application of the analytical model to acoustic foams with rather low airflow resistivity if the HR has to be tuned at low frequencies (note: this is usually the needed case).

From the homogenization method, Boutin derives effective properties for the resonant material. ${ }^{10}$ The effective density $\rho_{\text {eff }}$ is the one of the porous matrix $\rho_{\text {eq }}$ corrected by a factor $A$ to account for the macroscopic permeability decrease due to the presence of rigid inclusions: $\rho_{\mathrm{eff}}=\rho_{\mathrm{eq}} / A$ with $A \approx 2(1-c) /(2+c)$, where $c$ is the ratio of the HR volume to the PUC volume. The bulk modulus of the porous matrix $K_{\text {eq }}$ is highly impacted by the presence of the acoustic resonators and the effective property of the equivalent media is given by

$$
K_{\text {eff }}^{-1}=K_{0}^{-1}\left((1-c) \frac{K_{0}}{K_{\mathrm{eq}}}+\frac{c \omega_{0}^{2}}{\omega_{0}^{2}-\omega^{2} \tau^{\prime}}\right),
$$

where $K_{0}$ is the adiabatic bulk modulus of air, $\tau^{\prime}$ is the dynamic tortuosity function of the air layer in the HR neck calculated from Ref. 20 to account for losses in the resonator. Under the large wavelength assumption and by neglecting the viscous dissipations within the neck, this frequency can be approximated by $f_{0}=c_{0} / 2 \pi\left(S_{n} / V\left(d_{n}+\varepsilon_{i}+\varepsilon_{e}\right)\right)^{0.5}$ where $V$ is the volume of the resonator cavity. Finally, the transfer matrix of the homogenized equivalent material can be simply calculated from Eq. (2) but $Z_{\text {eq, } i}$ has to be replaced by the effective characteristic impedance $Z_{\text {eff }}=\left(\rho_{\text {eff }} \cdot K_{\text {eff }}\right)^{1 / 2}$, $k_{\text {eq }, i}$ by the effective wave number $k_{\text {eff }}=\omega\left(\rho_{\text {eff }} / K_{\text {eff }}\right)^{1 / 2}$ and $l_{i}$ by $l$. Equations (10)-(12) can then be used to compute its normal incidence acoustical properties.

\section{E. Numerical model}

An FE model based on the commercial code COMSOL Multiphysics $\left(\mathrm{COMSOL}^{\circledR}\right.$, Sweden) is used to validate the 1D acoustical behavior of one resonant unit cell predicted by the proposed P-TMM approach. The numerical model reproduces impedance tube measurements as shown in Fig. 3(a). A unit pressure is applied on the source side to simulate the normal incidence excitation. From the virtual measurements (a)

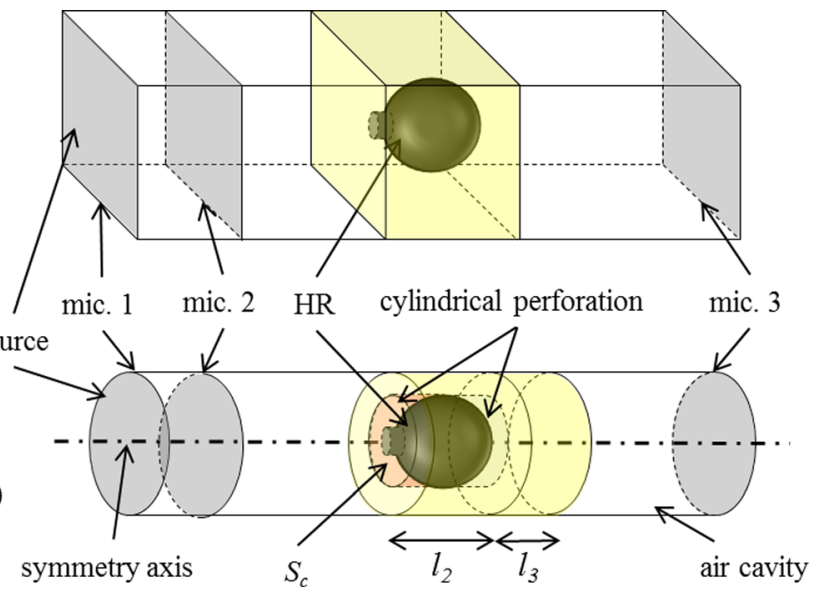

FIG. 3. (Color online) FE model of the impedance tube configuration: (a) cubic PUC; (b) cylindrical PUC with a cylindrical perforation in layer 2.

of the acoustic pressure at the three microphone locations, normal incidence sound absorption coefficient and sound transmission loss are deduced according to the three microphone method ${ }^{24}$ and standard ASTM E2611-09. ${ }^{25}$

The unit cell modeled numerically is either cubic[see Fig. 3(a)] or cylindrical [see Fig. 3(b)]. The HR walls are modeled as impervious, rigid, and motionless. The JohnsonChampoux-Allard equivalent fluid rigid model is used for the foam matrix and for the air layer situated in the HR neck to account for viscous and thermal dissipations. The effective properties of the air in the HR neck are computed using a cylindrical pore model. ${ }^{16}$ The properties of all equivalent fluids used in this work are provided in Table I. The cubic cell respects the periodicity boundary condition and will be used to validate the P-TMM model by comparison with the homogenization model in the case of a HR fully embedded in a low airflow resistivity open-cell foam.

Numerical simulations of a cylindrical unit-cell [see Fig. 3(b)] will be compared to P-TMM simulations and experiments carried out in the $100 \mathrm{~mm}$ cylindrical tube (see Sec. III A). In this case, since both the impedance tube and the HR neck are cylindrical and the HR cavity is spherical, the PUC is modeled in $2 \mathrm{D}$ considering a rotational symmetry. This decreases considerably the computation time compared to a 3D model. In this case, the HR is partially embedded in a medium airflow resistivity melamine foam for which layer 2 is perforated in its center in order to maintain the resonator. Since the proposed P-TMM model does not take into account

TABLE I. Material properties.

\begin{tabular}{lccc}
\hline \hline & $\begin{array}{c}\text { Open cell } \\
\text { foam (Ref. 10) }\end{array}$ & Melamine & $\begin{array}{c}\text { Neck of HR1 } \\
\text { for FE }\end{array}$ \\
\hline Porosity $\phi$ & 0.8 & 0.99 & 1 \\
Airflow resistivity $\sigma\left(\mathrm{N} \mathrm{s} \mathrm{m}^{-4}\right)$ & 298 & 7300 & 7 \\
Tortuosity $\alpha_{\infty}$ & 1 & 1 & 1 \\
Viscous characteristic & 500 & 88 & $4.6 \times 10^{3}$ \\
length $\Lambda(\mu \mathrm{m})$ & 800 & 160 & $4.6 \times 10^{3}$ \\
Thermal characteristic & & & 1.2 \\
length $\Lambda^{\prime}(\mu \mathrm{m})$ & - & 5.5 & \\
Density $\rho_{1}\left(\mathrm{~kg} \mathrm{~m}{ }^{-3}\right)$ & & & \\
\hline \hline
\end{tabular}


the air layer inside the spherical perforation (made to host the HR) above the HR front face [see Fig. 2(b)], the FE simulations are carried out with two different conditions at the perforation/foam interface $S_{c}$ as shown in Fig. 3(b): this interface is either considered impervious and rigid or fully permeable (non-existing). The FE configuration where $S_{c}$ is rigid and impervious is referred to as the semi/open configuration.

\section{EXPERIMENTAL SETUPS AND MATERIALS}

\section{A. Experimental setups}

The normal incidence sound absorption coefficient $\alpha$ is measured according to standard ASTM E1050-10 (Ref. 26) using a cylindrical $100 \mathrm{~mm}$ diameter impedance tube [see Fig. 4(a)] and a square $30.5 \mathrm{~cm} \times 30.5 \mathrm{~cm}$ cross section impedance tube [see Fig. 4(b)]. The normal incidence sound transmission loss $T L_{n}$ is determined from pressure measurements at three positions within the impedance tube according to the three microphone method ${ }^{24}$ and standard ASTM E2611-09. ${ }^{25} T L_{n}$ measurements are only carried out in the $100 \mathrm{~mm}$ cylindrical impedance tube.

Diffuse field transmission loss tests of a large curve composite panel covered by different resonant sound packages were carried out at GAUS using a hemi-anechoic-reverberant transmission loss suite [see Figs. 4(c) and 4(d)]. The structure size was $161.5 \mathrm{~cm} \times 161.5 \mathrm{~cm}$. The intensity technique is used to determine the transmission loss. The technique follows closely standard ISO $15186-1 .^{27}$ The reverberation chamber (volume $\approx 143 \mathrm{~m}^{3}$ ) is excited using six loudspeakers and sound power is measured using a rotating microphone boom. On the anechoic side, the sound intensity is measured using an intensity probe. To cover the 100 to $5000 \mathrm{~Hz}$ frequency range, a $12 \mathrm{~mm}$ spacer along with $1 / 4$ in. microphones are used.

The diffuse field sound absorption coefficient of the resonant sound packages is measured according to Standard ASTM C423. ${ }^{28}$ The test procedure follows closely the aforementioned standard apart from the size of the tested article. Indeed the tested samples were those tested in the transmission loss facilities and thus were only $161.5 \mathrm{~cm} \times 161.5 \mathrm{~cm}$. Samples were installed on the room's floor as shown in Fig. 4(f). A wooden frame of $101.6 \mathrm{~mm}$ high (or $114.3 \mathrm{~mm}$ depending on the configurations) was taped on the material sides to avoid lateral absorption [see Figs. 4(e) and 4(f)]. Sabine absorption coefficients were calculated following the standard procedure.

\section{B. Materials}

Three main sound packages are investigated in this work and are based on large cubic PUC $(l \approx L \approx 100 \mathrm{~mm})$ with embedded spherical resonators tuned to $380 \mathrm{~Hz}$ (referred to as HR1) or $150 \mathrm{~Hz}$ (referred to as HR2). The geometrical properties of the embedded HRs are given in Table II. The cavity of HR1 and HR2 is spherical and made of plastic Christmas ornaments and the neck is a custommade plastic cylinder. The neck of HR 1 is $7 \mathrm{~mm}$ long and penetrates slightly into the HR cavity for about $2 \mathrm{~mm}$. HR2 has a cavity radius of $40 \mathrm{~mm}$ which makes it relatively compact and its low frequency resonance (i.e., $150 \mathrm{~Hz}$ ) is obtained by using a $65 \mathrm{~mm}$ long neck which enters into the cavity for about $60 \mathrm{~mm} .{ }^{29}$ Note that the mass of the used HRs have not been optimized and work is currently undertaken to improve the lightness of the sound package.

The first sound package was not tested experimentally. It is made from resonator HR1 fully embedded in low flow resistivity open-cell foam taken from Ref. 10. The PUC is cubic with a side length of $l=L=88.6 \mathrm{~mm}$ and the material respects the requirements for co-dynamic. It will be investigated in Sec. IV A 1 for comparison purposes of the proposed P-TMM with the homogenization based model. The airflow resistivity and tortuosity properties of the porous substrate are taken from Ref. 10. The two characteristic lengths (not provided in Ref. 10) have been chosen relatively large to be consistent with fully reticulated and low flow resistivity foams having large cells. ${ }^{30}$

The other two types of sound packages were fabricated and tested to validate the proposed model. They use
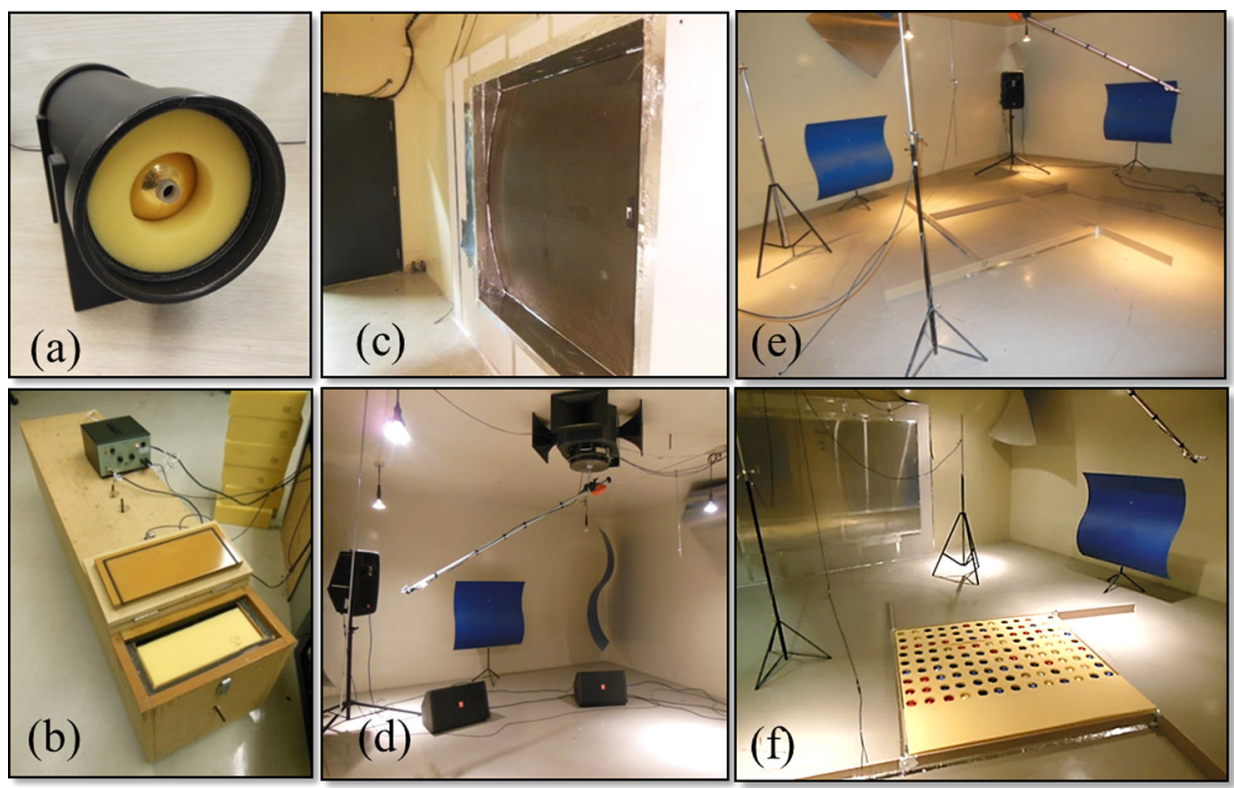

FIG. 4. (Color online) Pictures of the experimental setups: (a) cylindrical impedance tube; (b) large square impedance tube. (c) and (d) transmission loss test facility and curved composite panel. (e) and (f) diffuse field sound absorption test facility: (e) empty room, (f) room with sound package. 
TABLE II. Spherical HR properties.

\begin{tabular}{lcc}
\hline \hline & HR1 & HR2 \\
\hline Resonance frequency $f_{0}(\mathrm{~Hz})$ & 383 & 144 \\
Hollow sphere inner radius $(\mathrm{mm})$ & 29 & 39 \\
Neck depth $d_{n}(\mathrm{~mm})$ & 7 & 65 \\
Neck inner radius $R_{n}(\mathrm{~mm})$ & 4.6 & 6.3 \\
Mass $M(\mathrm{~g})$ & 7.4 & 22.3 \\
\hline \hline
\end{tabular}

melamine as host foam for resonators HR1 or HR2. The non-acoustical properties of this medium flow resistivity foam are obtained by the methods described in Ref. 31 and are provided in Table I. For practical reasons related to material fabrication, the melamine matrix is made of three layers: layer 2 is $76.2 \mathrm{~mm}$ thick, layer 3 (when used) is $25.4 \mathrm{~mm}$ thick, and layer 1 (when used) is $12.7 \mathrm{~mm}$ thick. The inner porous layer of thickness $l_{2}$ is perforated in order to host the spherical HR. The diameter of the cylindrical perforation is slightly inferior to the HR cavity outer diameter to ensure the position of the HR inside the foam (since no adhesive is used).

Various resonant materials were fabricated as shown in Fig. 5 and their detailed description is provided in Sec. IV. One cylindrical resonant unit-cell with partially embedded HR1 is measured in the $100 \mathrm{~mm}$ impedance tube [see Fig. 5(a)] whereas resonant materials made of four cells are tested in the square tube [see Fig. 5(b)]. Large sound packages are also fabricated and tested under diffuse acoustic field [see Figs. 5(c)-5(h)]. They are made of 156 resonant PUCs of surface $116.7 \mathrm{~mm} \times 124.4 \mathrm{~mm}$, to give a surface ratio of $r=19.5 \%$ (respectively, $r=34.6 \%$ ) when resonators HR1 (respectively, HR2) are installed. These sound packages were designed to cover the entire composite panel surface $(161.5 \mathrm{~cm} \times 161.5 \mathrm{~cm})$ during the transmission loss tests. Thus, they are split into three different pieces to simplify its installation on the curved panel as shown in Fig. $5(\mathrm{~g})$. The three flat pieces were simply laid on the panel surface and neither glue nor tape was used. A thin air layer is thus created between the planar resonant material and the curved panel. In order to investigate the impact of the orientation of the resonators neck on the acoustical properties of the resonant material, the sound packages will be tested in both positions $\mathbf{A B}$ (i.e., for an acoustic wave impinging on the neck side of the HR) and BA (i.e., for an acoustic wave impinging on the rear side of the HR) as illustrated in Fig. $5(\mathrm{~g})$ in the case of the transmission loss tests.

\section{RESULTS}

\section{A. Validation of the one dimensional P-TMM model}

The normal incidence behavior of the resonant material including fully or partially embedded resonators HR1 or HR2 is investigated both analytically (P-TMM, homogenization model), numerically (FE), and experimentally.

\section{One PUC with fully embedded HR}

The P-TMM, homogenization, and FE methods are first applied to the material made of the resonator HR1 fully embedded in the low flow resistivity open-cell foam [see configuration of Fig. 3(a)]. The resonant material respects all the requirements for a co-dynamic regime: (1) the neck section $S_{n}$ is much smaller than that of the period $S_{n} / L^{2}=8.5 \times 10^{-3}$ $\ll 1$ and (2) the HR resonance occurs at a HR resonant frequency $f_{0}$ corresponding to the inertial regime of the host foam, i.e., $f_{0} / f_{c}=12 \gg 1$ where $f_{c}$ is the viscous/inertial transition frequency which can be approximated by $f_{c}=\phi \sigma /$ $\left(2 \pi \alpha_{\infty} \rho_{0}\right)$. The resonant PUC is cubic with a side length of $L=88.6 \mathrm{~mm}$. In the case of the proposed P-TMM modeling, the PUC is made of three main layers. Layers 1 and 3 are $15.3 \mathrm{~mm}$ thick and layer 2 which carries HR 1 is $58 \mathrm{~mm}$ thick (i.e., the diameter of the spherical resonator). Layer 2 is then subdivided in two layers. Layer $2_{\mathrm{a}}$ in this case is homogeneous and made of the same material as the foam matrix since HR1 is completely buried within the foam. The surface ratio in layer $2_{\mathrm{HR}}$ is $r=33.6 \%$ and the resonator concentration in the PUC is $c=0.15$.

Figures 6(a), 6(b), and 6(c) present, respectively, the normal incidence sound absorption coefficient of the PUC in position AB and $\mathbf{B A}$ backed by a $25.4 \mathrm{~mm}$ air cavity and its normal incidence sound transmission loss. Compared to a homogeneous foam of identical thickness (see solid gray curves in Fig. 6), the resonant material shows a sound absorption and transmission peak with high amplitude in a narrow frequency band around the HR resonance frequency (i.e., $380 \mathrm{~Hz}$ ). For such material made up of acoustic resonators, these peaks are related to a negative group velocity and negative bulk modulus of the equivalent metamaterial. ${ }^{7-9,14}$ Indeed for wavelengths larger than the dimension of the

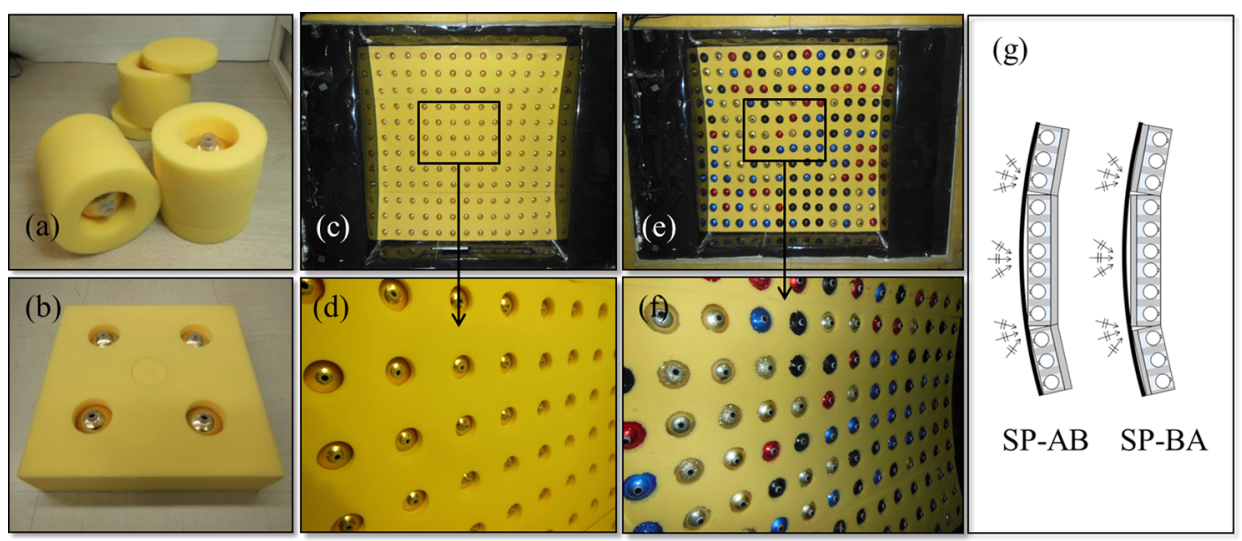

FIG. 5. (Color online) Pictures of the tested resonant materials: (a) cylindrical PUC made of HR1; (b) four squared PUC made of HR1; (c) and (d) sound package with $156 \mathrm{HR} 1 \mathrm{~s}$; (e) and (f) sound package with $156 \mathrm{HR} 2 \mathrm{~s}$; (g) schematic of the tested configurations: SP-AB, the HRs face the panel, SPBA, the HRs face the hemi-anechoic room. 


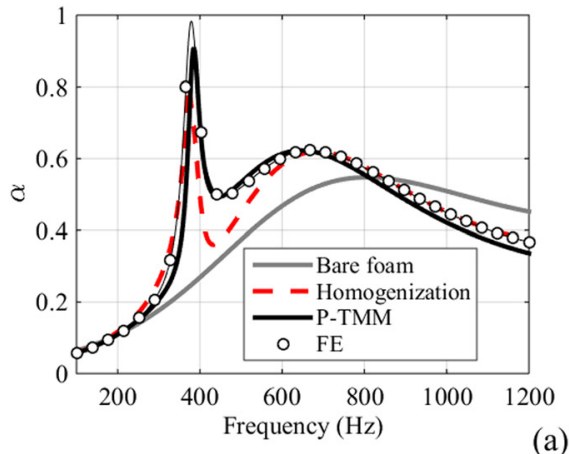

(a)

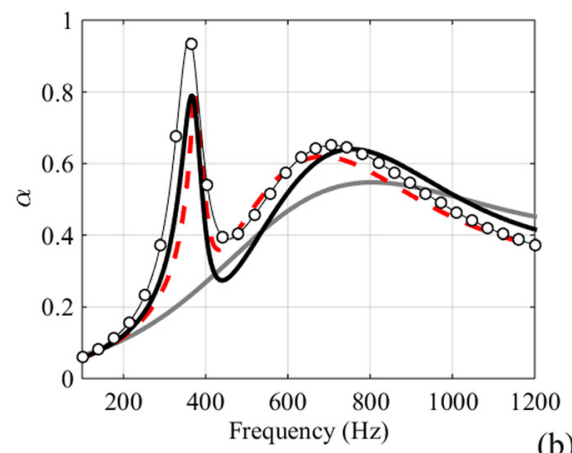

(b)

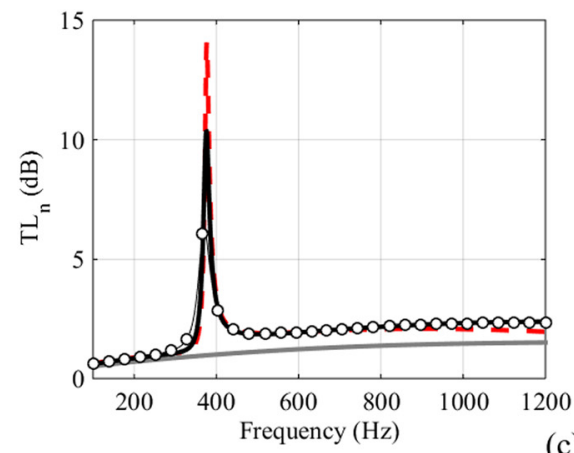

(c)

FIG. 6. (Color online) P-TMM versus homogenization (Ref. 10) and FE models in the case of a cubic PUC with fully embedded HR1; (a) sound absorption coefficient of the PUC in position AB when backed by a $25.4 \mathrm{~mm}$ thick air cavity; (b) sound absorption coefficient of the PUC in position BA when backed by a $25.4 \mathrm{~mm}$ thick air cavity, and (c) normal incidence sound transmission loss.

PUC, the system behaves as an effective medium. The wave transport is then controlled by effective density and effective bulk modulus. In this case, the local resonance makes the bulk modulus negative and propagation is impeded. Around local resonances of the HR, the response function has large dispersion, leading to a dip in the region of negative effective bulk modulus and hence exponential wave attenuation.

According to Fig. 6, the global simulated trend from low to mid frequencies by the three modeling strategies is in fairly good agreement. The main difference between all methods is the prediction around and just above the frequency of the peaks.

Both FE and P-TMM modeling strategies allow to observe an asymmetry on the normal incidence sound absorption coefficient between the AB and BA PUC positions: the sound absorption peak is slightly lower in amplitude and in frequency in the BA position and the sound absorption dip for frequencies just above the peak is also more pronounced in the BA position. Considering the FE simulations as reference, it can be observed that the P-TMM better predicts the PUC sound absorption behavior in the $\mathbf{A B}$ position compared to the $\mathbf{B A}$ position. This can be attributed to the geometrical considerations in the modeling of the inner layer 2, i.e., the definition of the thicknesses of the sublayers $l_{2 a}$ and $l_{2 \mathrm{HR}}$. Nevertheless, the asymmetry observed between positions $\mathbf{A B}$ and $\mathbf{B A}$ and captured by the P-TMM strategy indicates that the proposed modeling strategy is adapted for such resonant material having $2 \mathrm{D}$ periodicity. It has to be noted that the homogenization model has been derived in Ref. 10 for a 3D periodicity, while in this work, the periodicity is only 2D: the loss of periodicity in one direction might be responsible for some local phenomena observed, such as the sensitivity of results to the resonator orientation.

\section{One PUC with partially embedded HR}

The proposed P-TMM model is now applied to one unit cell made of HR1 embedded in the medium flow resistivity melamine foam. The co-dynamic regime is not fulfilled in this case since the HR resonance occurs at a frequency $f_{0}$ corresponding to the viscous regime of the host foam, i.e., $f_{0} / f_{c}=0.39<1$. HR 1 is thus partially embedded in the porous matrix in order for the neck to be in direct contact with an air layer. This configuration can be simulated with the P-TMM strategy by simply removing layer 1 as detailed in Sec. II A. The foam matrix of the PUC is thus made of layers 2 and $3(l=101.6 \mathrm{~mm})$ and includes the resonator HR1 in the center of layer 2 [see Figs. 3(b), 4(a), and 5(a)]. The surface ratio in this case is $r=36 \%$. The PUC is cylindrical and $100 \mathrm{~mm}$ in diameter to be measured in the impedance tube. Measurements, P-TMM, and FE simulations are presented in Fig. 7. P-TMM predictions are performed with both the rigid frame and the limp frame model assumptions for the porous matrix. ${ }^{16}$ The normal incidence coefficients shown in Fig. 7 (see first line) are measured when the PUC is backed by a $25.4 \mathrm{~mm}$ air cavity. The gray surface delimits the minimum and maximum values measured for three different samples.

Figures 7(a) and 7(b) present the acoustic features for a $101.6 \mathrm{~mm}$ thick melamine sample (no HR, no perforation). It is shown that both the rigid and limp assumptions correctly estimate the sound absorption behavior. However, the limp assumption should be preferred to predict the transmission behavior of the porous matrix.

The HR is now inserted in the perforated porous matrix and the acoustic behavior of the PUC is measured either in position AB [see Figs. 7(c) and 7(d)] or BA [see Figs. 7(e) and 7(f)]. Three dips can be observed in the measured sound absorption curves. The first one, occurring around $220 \mathrm{~Hz}$, and the third one, occurring around $1200 \mathrm{~Hz}$, are attributed to mechanical resonances of the porous matrix. They appear only when the HR is present since the perforation is slightly smaller than the HR diameter and the HR exerts a lateral compression on the foam matrix against the impedance tube's wall. The material is thus artificially stiffened and the excitation of the porous frame is favored. These two mechanical resonances also affect the transmission loss as shown in Figs. 7(d) and 7(f). Both P-TMM and FE simulations do not predict these dips related to mechanical resonances since the equivalent fluid assumption is used to model the foam matrix. Furthermore, these resonances highly depend on the boundary and mounting conditions of the sample and do not characterize the behavior of the cellular material in more realistic conditions. As explained previously, the HR insertion exerts a lateral compression and 


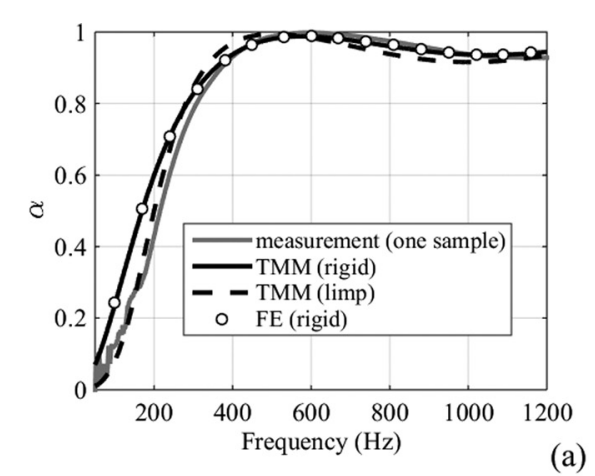

(a)

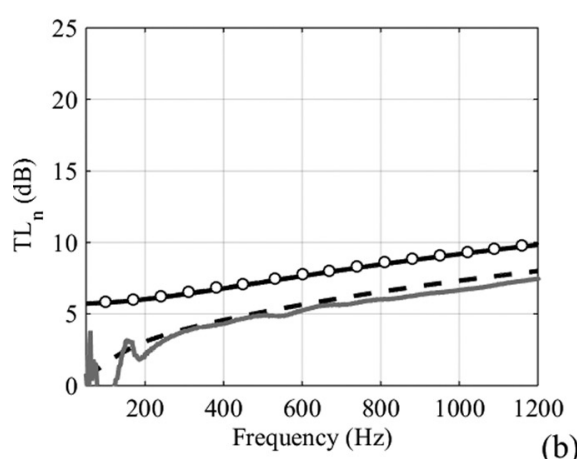

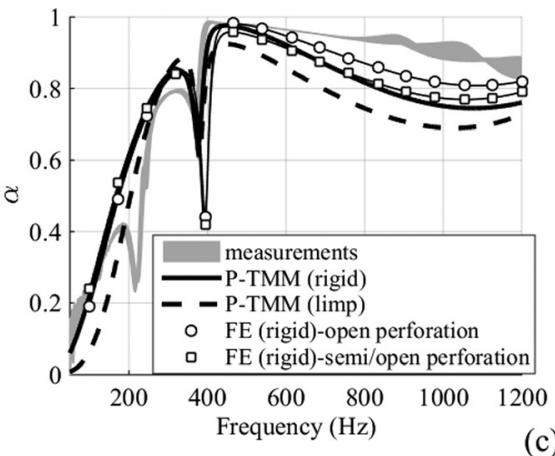

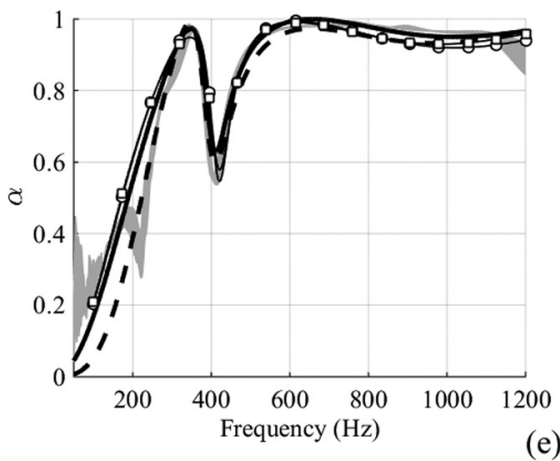

(c)

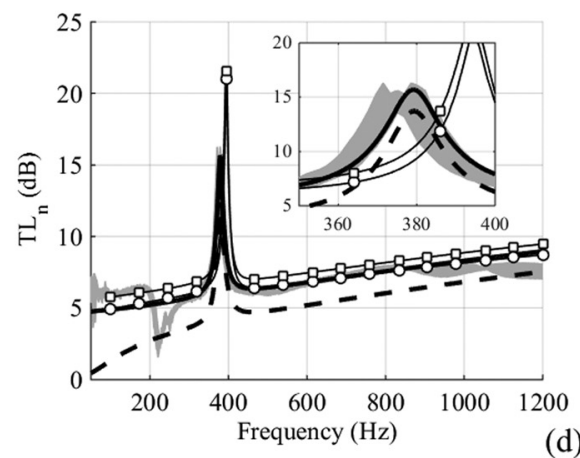

FIG. 7. (First line) Normal incidence sound absorption coefficient and (second line) normal incidence sound transmission loss of 1 cylindrical PUC: (first column) bare melamine; (second column) PUC in position AB; (third column) PUC in position BA.

stiffens artificially the material. This is the reason why the rigid frame model better captures the acoustic properties of the resonant PUC [Figs. 7(d) and 7(f)].

The second sound absorption dip occurs around the HR resonance frequency $f_{0}$ (i.e., $375 \mathrm{~Hz}$ ) in position $\mathbf{A B}$ and slightly above $f_{0}$ (i.e., $400 \mathrm{~Hz}$ ) in position BA. It can be observed that the sound absorption dip is more pronounced in the latter position. Above $500 \mathrm{~Hz}$, the sound absorption efficiency in position $\mathbf{B A}$ is greater than the one in position AB. Furthermore, a decrease of the sound absorption efficiency of the PUC at the HR resonance is quite unexpected since HRs are usually used to improve this acoustic property. The analysis of this negative effect is out of the scope of the present paper and will be presented in a forthcoming paper. Both the sound absorption dip and sound transmission peak associated to the HR resonance frequency are correctly predicted by the P-TMM in both positions AB and BA. The amplitude of the dip (respectively, peak) in the sound absorption curve (respectively, sound transmission loss curve) at the $\mathrm{HR}$ resonance is more pronounced in the $\mathrm{FE}$ results compared to P-TMM and is also slightly shifted toward higher frequencies. Since the P-TMM successfully simulates the measured acoustic features at the HR resonance frequency, the differences between P-TMM and FE at this frequency is more likely due to an inaccuracy in the FE simulation. However, this was not investigated.

The main discrepancy between measurements and P-TMM simulations arises in the sound absorption coefficient in position $\mathbf{A B}$ for frequencies just above $f_{0}$ where the P-TMM underestimates the sound absorption coefficient. In this case, the FE simulation is in better agreement with P-TMM above $400 \mathrm{~Hz}$ when surface $S_{c}$ is replaced by an impervious wall [see white squares in Fig. 7(c)]. This indicates that the underestimation of the sound absorption in position AB observed between P-TMM and impedance tube measurements can partly be attributed to sound absorption at the perforation walls in front of the HR. The semi/close FE configuration is also associated to a slight increase in sound transmission loss $(<1 \mathrm{~dB})$. This is expected since more acoustic energy has been reflected at the upstream face of the PUC.

\section{Four PUCs with partially embedded HR}

The normal incidence sound absorption of hard-backed materials made of four square resonant PUCs measured in the large tube are presented in Fig. 8. The materials are made only of the inner layer 2 (i.e., $l=l_{2}=76.2 \mathrm{~mm}$ ) and the resonators HR1 [see Fig. 8(b)] or HR2 [see Fig. 8(c)] are inserted in the melamine foam substrate. The side of the PUC is $L=152 \mathrm{~mm}$. The surface ratio is now $r=13 \%$ for HR 1 material and $r=22 \%$ for HR 2 material. It is shown that resonator HR1 now has a positive contribution to the sound absorption of the melamine matrix at the HR resonance frequency. This opposite behavior compared to previous results shown in Fig. 7(c) is not due to the greater number of HRs embedded in the material but is rather due to the thinner porous matrix $(76.2 \mathrm{~mm}$ vs $101.6 \mathrm{~mm})$ and the decrease of the surface ratio. The sound absorption behavior of the material with HR2 is greatly improved at the HR resonance frequency. It is observed that the P-TMM model correctly predicts the effect of the embedded HR for the two materials. 

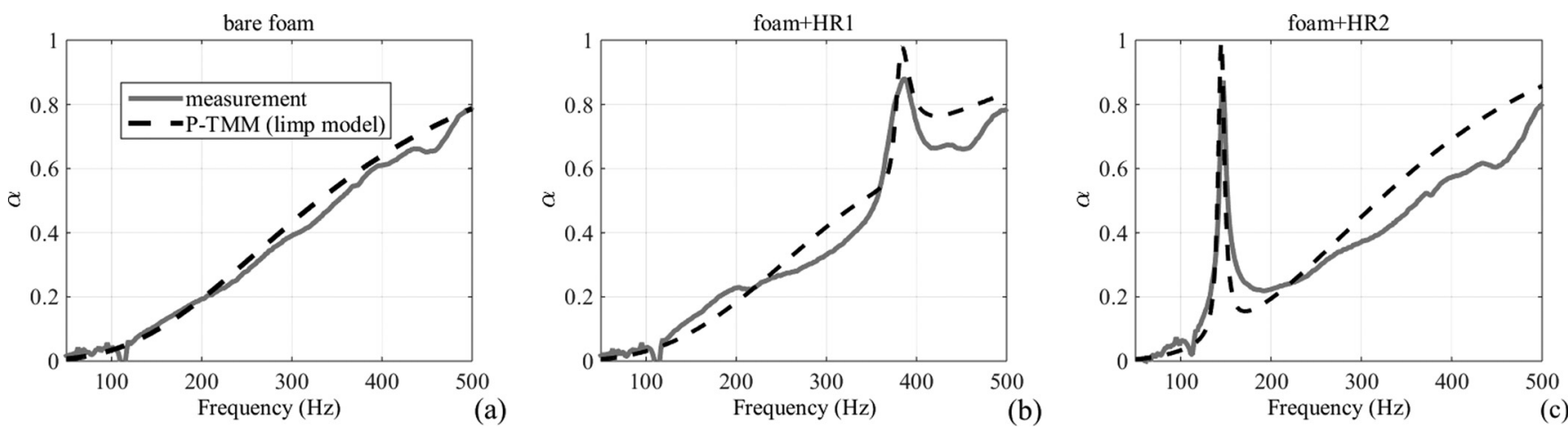

FIG. 8. Normal incidence sound absorption coefficient of rigid backed resonant materials made of four squared PUCs. Porous frame: 76.2 mm melamine. PUC size: $152 \mathrm{~mm} \times 152 \mathrm{~mm}$. (a) Bare foam, (b) $4 \times \mathrm{HR} 1 \mathrm{~s}$, and (c) $4 \times \mathrm{HR} 2 \mathrm{~s}$.

\section{B. Large scale measurements}

\section{Transmission loss measurements of a large composite curved panel}

The diffuse field transmission loss of a composite curved panel covered by the resonant sound package is measured. Two main HRs sound packages are investigated; ones filled with resonators HR1 and others filled with resonators HR2. As detailed in Sec. III, all large scale sound-packages are made with melamine foam: layer 3 is $25.4 \mathrm{~mm}$ thick, layer 2 is $76.2 \mathrm{~mm}$ thick, and layer 1 (when used) is $12.7 \mathrm{~mm}$ thick. The parameters of the sound packages are detailed in Table III. Note that the sound package density has almost doubled (respectively, quadrupled) in the case of HR1 (respectively, HR2) when compared to the bare foam material. Even if a density of $10 \mathrm{~kg} \mathrm{~m}^{-3}$ can be acceptable, much effort still has to be done to lighten the proposed resonant structure.

Figure 9 presents the insertion loss of the tested sound packages. It is calculated considering the "plate/bare foam" structure as the baseline configuration: $I L=T L_{\text {conf }}-T L_{\text {baseline }}$. The thickness of the bare foam used in the baseline configuration is $101.6 \mathrm{~mm}$. Figure 9 shows that there is no noticeable change of the sound transmission loss before the resonance $f_{0}$ (i.e., $I L$ close to $0 \mathrm{~dB}$ ). But, the transmission loss improvement at $f_{0}$ observed for normal incidence excitation is confirmed for diffuse field excitation. A maximum IL of $6.6 \mathrm{~dB}$ is found at the 12th octave band centered at $355 \mathrm{~Hz}$ in the case of sound package HR1 [see Fig. 9(a)]. The efficient bandwidth (BD), defined here as the frequency bandwidth around $f_{0}$ for which the IL exceeds $3 \mathrm{~dB}$, is relatively large and approximates $60 \mathrm{~Hz}$. In the case of sound package HR2 [see Fig. 9(b)], the maximum IL is around $8.5 \mathrm{~dB}$ in the 12 th octave band centered

TABLE III. Sound package configurations for the large scale experiments. PUC surface $=116.7 \times 124.4 \mathrm{~mm}^{2}$

\begin{tabular}{lcccc}
\hline \hline Configuration name & SP1 & SP2 & SP3 & SP4 \\
\hline HR type & HR1 & HR1 & HR2 & HR2 \\
Perforation diameter $(\mathrm{mm})$ & 60 & 60 & 80 & 80 \\
HR surface ratio $r(\%)$ & 19.5 & 19.5 & 34.6 & 34.6 \\
Layer 1 thickness $(\mathrm{mm})$ & 0 & 12.7 & 0 & 12.7 \\
Overall thickness $l(\mathrm{~mm})$ & 101.6 & 114.3 & 101.6 & 114.3 \\
Equivalent density $\left(\mathrm{kg} \mathrm{m}^{-3}\right)$ & 9.7 & 9.2 & 19.2 & 17.7 \\
\hline \hline
\end{tabular}

at $150 \mathrm{~Hz}$ and the efficient bandwidth is $25 \mathrm{~Hz}$. The good transmission loss performance at the HR resonance frequency of the composite panel coupled to sound package HR2 can be attributed to the combination of two positive contributions of the sound package: (1) the sound transmission loss peak and (2) the sound absorption peak; the latter dampens the multiple acoustic wave reflections in the inner air layer between the panel and the material, as commented in Ref. 32. Above $f_{0}$, the performance of the resonant sound package depends on the configuration. However, one can observe an IL decrease of $2 \mathrm{~dB}$ around 400 and $700 \mathrm{~Hz}$ for all configurations and more particularly around the two $1 / 12$ octave bands centered on 500 and $630 \mathrm{~Hz}$. Two local transmission loss minima at these frequencies were already observed on the transmission loss of the bare panel and thus can be attributed to structural resonances. The sound package with the resonant inclusions (and perforations) thus seems less efficient at dissipating the vibrational energy at these frequencies when compared to the bare foam material. Note that the added mass due to the presence of the resonators has no impact on the transmission loss of the structure since it remains relatively negligible compared to the mass of the panel.

When comparing sound packages SP1 (respectively, SP3) and SP2 (respectively, SP4), it is observed that better results are achieved when the HR is covered by the porous layer 1 (i.e., SP2 and SP4 configurations): e.g., the IL always remains above $0 \mathrm{~dB}$ in the case of SP2 and the good performance at $f_{0}$ for both SP2 and SP4 is preserved (i.e., IL peak around $5 \mathrm{~dB}$ ). It is worth noting that the HR of configurations SP2 and SP4 cannot be considered as completely embedded in the porous substrate since it is placed within the cylindrical perforation and its neck is in contact with air and not surrounded by the porous material as presented schematically in Fig. 3(a).

Positions $\mathbf{A B}$ and $\mathbf{B A}$ are now compared and the asymmetry observed in the case of the $1 \mathrm{D}$ acoustic behavior is confirmed. For all configurations, it is found that the peak amplitude is higher in the AB position (except for SP4) and that the IL above $f_{0}$ is higher for the position BA. These two phenomena have to be related to the combination of the sound package transmission loss and sound absorption in the air cavity as described in Ref. 32. Indeed, both positions give identical transmission loss for all frequencies as shown in Fig. 7 but (1) position $\mathbf{A B}$ provides the better sound 

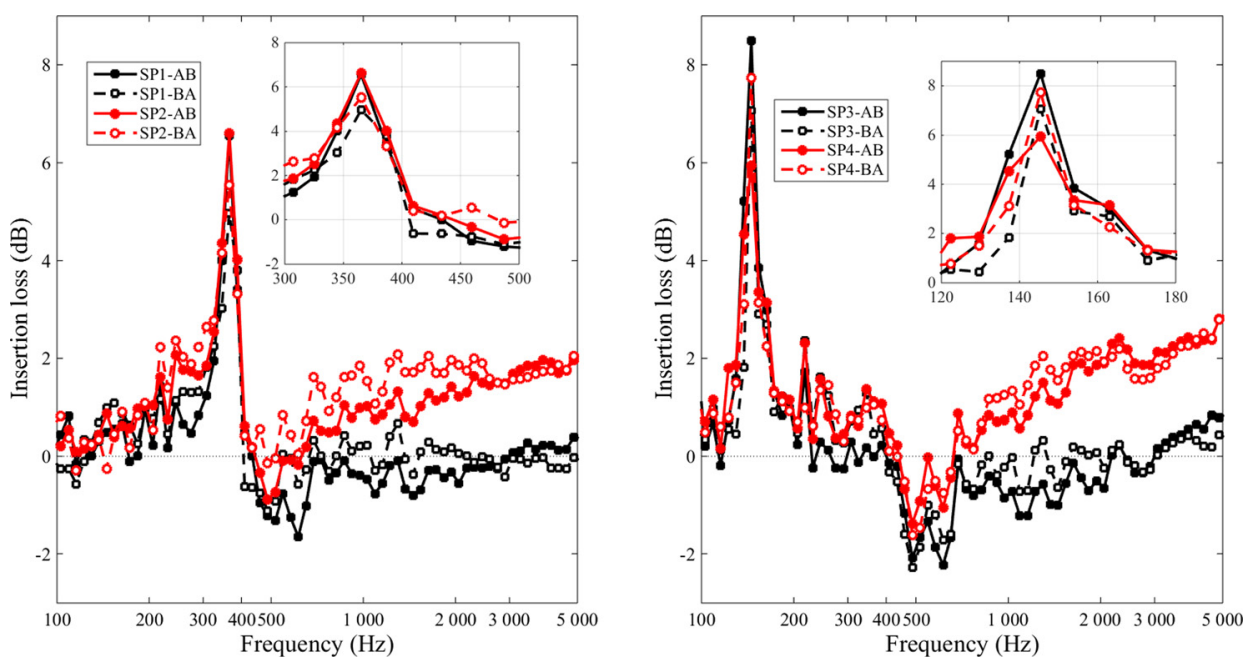

FIG. 9. (Color online) Diffuse field insertion loss measurements $(101.6 \mathrm{~mm}$ thick melamine alone taken as reference) of the curved panel covered by the resonant sound packages in position AB or BA with $156 \mathrm{HRs}$ tuned to (a) $380 \mathrm{~Hz}$; (b) $145 \mathrm{~Hz}$.

absorption efficiency at the HR resonance frequency $f_{0}$ and (2) position BA provides the better sound absorption efficiency above this frequency.

\section{Diffuse field sound absorption coefficient}

Only the sound package SP4 with the resonator HR2 has been tested in the diffuse absorption coefficient facility (the porous substrate of SP1 and SP2 was used to fabricate SP3 and SP4). Figure 10 presents the sound absorption coefficient for sound package SP4 in both positions $\mathbf{A B}$ and $\mathbf{B A}$. The main figure provides $1 / 3$ octave bands data whereas the inner box zoom in on the low frequency region (from 125 to $150 \mathrm{~Hz}$ ) and presents data in 1/12 octave bands. For these highly absorbing materials, the standard measurement procedure results in a non-physical absorption coefficient above $150 \mathrm{~Hz}$ for all configurations $(\alpha>1)$. This well-known behavior is due to the finite size of the sample and to the room effects. ${ }^{33-35}$

The 1/12 octave bands results presented in Fig. 10 clearly show an improvement of the material sound absorption at the HR resonance frequency. The large absorption

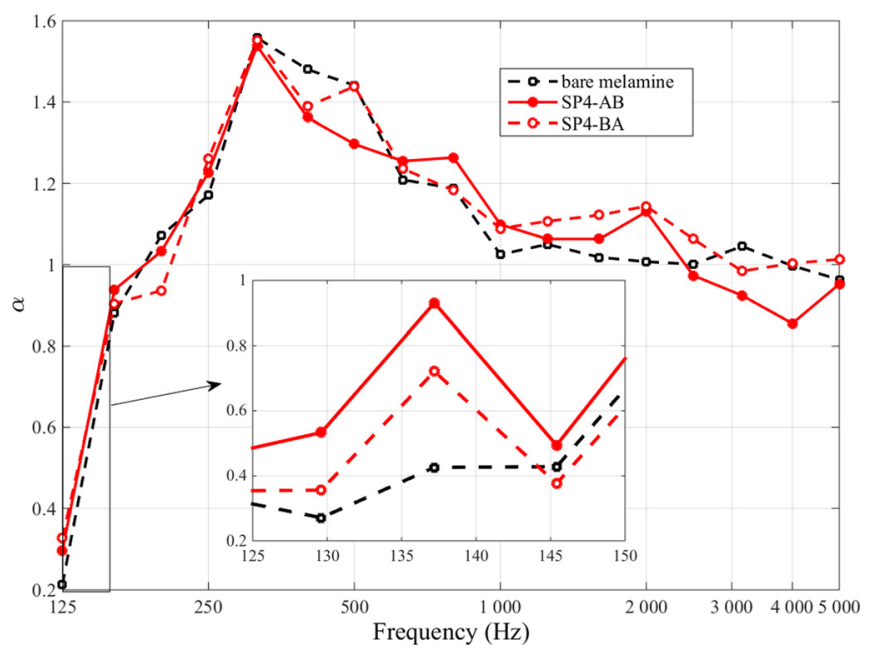

FIG. 10. (Color online) Diffuse field sound absorption coefficient of $101.6 \mathrm{~mm}$ thick bare melamine and of the sound package SP4 in position AB or BA. peak can be observed even when the HR is embedded in the foam material (because of the perforations as mentioned previously) and the HRs' neck faces the room floor (i.e., position BA). Two important effects observed in the normal incidence sound absorbing behavior of this resonant material [see Figs. 7(c) and 7(e)] either in position $\mathbf{A B}$ or $\mathbf{B A}$ are confirmed by the diffuse field measurements (see Fig. 10): (1) the sound absorption of the material in position $\mathbf{B A}$ is decreased compared to the bare material for frequencies just above $f_{0}$ (see the $1 / 3$ octave band centered at $200 \mathrm{~Hz}$ ) and (2) the sound absorption efficiency of the material in position $\mathbf{B A}$ is greater compared to the one in position $\mathbf{A B}$ from mid to high frequencies.

\section{CONCLUSION}

The acoustic properties of a cellular porous material with resonant inclusions have been investigated analytically, numerically, and experimentally. A model based on the TMM is proposed to model the normal incidence acoustic properties of the resonant material. The extended P-TMM used in this work allows assembling components in series (layers constituting the porous frame) and in parallel (inner porous frame layer with the resonant inclusion). It is shown that the transmission loss of the material is greatly improved at the HR resonance frequency. The HR contribution to the sound absorbing efficiency of the porous material can be positive or negative at this frequency and one should be careful when designing such material for application involving both sound absorption and sound transmission behavior (e.g., aircraft double wall structures, payload fairings of launch vehicles). Furthermore, it is shown that the sound absorbing behavior of the resonant material depends on the orientation of the HR neck either facing toward or away from the impinging acoustic wave. The P-TMM approach allows to correctly predict all these features and could be a useful tool in a first design phase. The flexibility of this model allows simulating fully and partially embedded resonators and thus opens the door for designing resonant material based on medium to high airflow resistivity foam substrate. Finally, large scale resonant materials including 156 HRs have been fabricated and tested under diffuse field 
excitation. The measured acoustic performances are in good agreement with those observed under normal incidence acoustic excitation. Furthermore, the frequency bandwidth around the HR resonance with a positive effect of the HR were found relatively large considering such poorly damped resonators as those used during the experiments. Future works include developing 3D numerical models to better assess the performance of the sound package under oblique and diffuse field incidence and taking into account the nonlinear behavior of the resonators when subjected to high sound pressure amplitude.

\section{ACKNOWLEDGMENTS}

The authors would like to thank the National Sciences and Engineering Research Council of Canada (NSERC) for providing financial support. We would like to thank Kevin Verdière, Thomas Dupont, and Raymond Panneton for helpful discussions during the course of this work. We also kindly thank Patrick Lévesque and Thomas Padois for their very useful help during the fabrication of more than 300 Christmas HRs. Finally, Maxime Bolduc and Raef Cherif are acknowledged for their contribution to the large scale measurements.

${ }^{1}$ I. U. Borchers, S. T. Laemmlein, P. Bartels, A. Rausch, M. Faust, J. A. F. Coebergh, and K. Koeble, "Acoustic protection on payload fairings of expendable launch vehicles," U.S. patent 5,670,758 (1997).

${ }^{2}$ W. O. Hughes and A. M. McNelis, "Cassini/Titan IV acoustic blanket development and testing," NASA Technical Memorandum 107266, prepared for the 42nd Annual Technical Meeting and Exposition sponsored by the Institute of Environmental Sciences, Orlando, FL (May 12-16, 1996).

${ }^{3}$ S. Sugie, J. Yoshimura, and T. Iwase, "Effect of inserting a Helmholtz resonator on sound insulation in a double-leaf partition cavity," Acoust. Sci. Tech. 30(5), 317-326 (2009).

${ }^{4}$ J. M. Mason and F. J. Fahy, "The use of acoustically tuned resonators to improve the sound transmission loss of double-panel partitions," J. Sound Vib. 124(2), 367-379 (1988).

${ }^{5}$ R. A. Prydz, L. S. Wirt, H. L. Kuntz, and L. D. Pope, "Transmission loss of a multilayer panel with internal tuned Helmholtz resonators," J. Acoust. Soc. Am. 87(4), 1597-1602 (1990).

${ }^{6}$ H. L. Kuntz, R. A. Prydz, and F. J. Balena, "Development and testing of cabin sidewall acoustic resonators for the reduction of cabin tone levels in prop fan-powered aircraft,” Noise Cont. Eng. J. 37(3), 120-142 (1991).

${ }^{7}$ C. L. Ding, L. Hao, and X. P. Zhao, "Two-dimensional acoustic metamaterial with negative modulus," J. Appl. Phys. 108, 074911 (2010).

${ }^{8}$ C. L. Ding and X. P. Zhao, "Multi-band and broadband acoustic metamaterial with resonant structures," J. Phys. D: Appl. Phys. 44, 215402 (2011).

${ }^{9} \mathrm{M}$. Reynolds and S. Daley, "An active viscoelastic metamaterial for isolation applications," Smart Mater. Struct. 23, 045030 (2014).

${ }^{10} \mathrm{C}$. Boutin, "Acoustics of porous media with inner resonators," J. Acoust. Soc. Am. 134(6), 4717-4729 (2013).

${ }^{11}$ C. Boutin and F.-X. Bécot, "Theory and experiments on poro-acoustics with inner resonators," Wave Motion 54, 76-99 (2015).

${ }^{12}$ C. Lagarrigue, J. P. Groby, V. Tournat, and O. Dazel, "Absorption of sound by porous layers with embedded periodic arrays of resonant inclusions," J. Acoust. Soc. Am. 134(6), 4670-4680 (2013).

${ }^{13}$ J.-P. Groby, C. Lagarrigue, B. Brouard, O. Dazel, V. Tournat, and B. Nennig, "Enhancing the absorption properties of acoustic porous plates by periodically embedding Helmholtz resonators," J. Acoust. Soc. Am. 137(1), 273-280 (2015).

${ }^{14}$ N. Fang, D. Xi, J. Xu, M. Ambati, W. Srituravanich, C. Sun, and X. Zhang, "Ultrasonic meta-material with negative modulus," Nat. Mater. 5, 452-456 (2006).

${ }^{15}$ W. Lauriks, P. Mees, and J. F. Allard, "The acoustic transmission through layered systems," J. Sound Vib. 155(1), 125-132 (1992).

${ }^{16}$ J. F. Allard and N. Atalla, Propagation of Sound in Porous Media: Modeling Sound Absorbing Materials, 2nd ed. (Wiley, Chichester, UK, 2009), Chap. 4, pp. 45-72; Chap. 5, pp. 73-107; Chap. 9.3.5, pp. 203-205; and Chap. 11, pp. 243-281.

${ }^{17}$ A. Pellicier and N. Trompette, "A review of analytical methods, based on the wave approach, to compute partitions transmission loss," Appl. Acoust. 68, 1192-1212 (2007).

${ }^{18}$ K. Verdière, R. Panneton, S. Elkoun, T. Dupont, and P. Leclaire, "Transfer matrix method applied to the parallel assembly of sound absorbing materials," J. Acoust. Soc. Am. 134(6), 4648-4658 (2013).

${ }^{19}$ K. Verdière, R. Panneton, S. Elkoun, T. Dupont, and P. Leclaire, "Comparison between parallel transfer matrix method and admittance sum method," J. Acoust. Soc. Am. 136(2), EL90-EL95 (2014).

${ }^{20}$ D. L. Johnson, J. Koplik, and R. Dashen, "Theory of dynamic permeability and tortuosity in fluid-saturated porous media," J. Fluid Mech. 176, 379-402 (1987)

${ }^{21}$ Y. Champoux and J. F. Allard, "Dynamic tortuosity and bulk modulus in air-saturated porous media," J. Appl. Phys. 70(4), 1975-1979 (1991).

${ }^{22}$ B. M. Efimtsov and L. A. Lazarev, "Sound transmission loss of panels with resonant elements," Acoust. Phys. 47(3), 346-351 (2001).

${ }^{23} \mathrm{R}$. Panneton, "Normal incidence sound transmission loss evaluation by upstream surface impedance measurements," J. Acoust. Soc. Am. 125(3), 1490-1497 (2009).

${ }^{24}$ Y. Salissou, R. Panneton, and O. Doutres, "Complement to standard method for measuring normal incidence sound transmission loss with three microphones," J. Acoust. Soc. Am. 131, EL216-EL222 (2012).

${ }^{25}$ ASTM E2611-09. Standard Test Method for Measurement of Normal Incidence Sound Transmission of Acoustical Materials Based on the Transfer Matrix Method (American Society for Testing and Materials).

${ }^{26}$ ASTM E1050-10. Standard Test Method for Impedance and Absorption of Acoustical Materials Using a Tube, Two Microphones and a Digital Frequency Analysis System (American Society for Testing and Materials).

${ }^{27}$ ISO 15186-1. Acoustics-Measurement of Sound Insulation in Buildings and of Building Elements Using Sound Intensity-Part 1: Laboratory Measurements (2000).

${ }^{28}$ ASTM C423-02. Standard Test Method for Sound Absorption and Sound Absorption Coefficient by the Reverberation Room Method (American Society for Testing and Materials).

${ }^{29}$ A. Selamet and I. Lee, "Helmholtz resonator with extended neck," J. Acoust. Soc. Am. 113(4), 1975-1985 (2003).

${ }^{30}$ O. Doutres, N. Atalla, and K. Dong, "A semi-phenomenological model to predict the acoustic behavior of fully and partially reticulated polyurethane foams," J. Appl. Phys. 113, 054901 (2013).

${ }^{31}$ O. Doutres, Y. Salissou, N. Atalla, and R. Panneton, "Evaluation of the acoustic and non-acoustic properties of sound absorbing materials using a three-microphone impedance tube," Appl. Acoust. 71, 506-509 (2010).

${ }^{32} \mathrm{O}$. Doutres and N. Atalla, "Acoustic contributions of a sound absorbing blanket placed in a double panel structure: Absorption versus transmission," J. Acoust. Soc. Am. 128(2), 664-671 (2010).

${ }^{33} \mathrm{~A}$. Nash, "On the reproducibility of measuring random incidence sound absorption," in Proceedings of Internoise 2012, New York, NY (August 19-22, 2012), pp. 1-12.

${ }^{34} \mathrm{C}$. H. Jeong, "Converting Sabine absorption coefficients to random incidence absorption coefficients," J. Acoust. Soc. Am. 133(6), 3951-3962 (2013).

${ }^{35}$ O. Robin, A. Berry, O. Doutres, and N. Atalla, "Measurement of the absorption coefficient of sound absorbing materials under a synthesized diffuse acoustic field,” J. Acoust. Soc. Am. 136(1), EL13-EL19 (2014). 